\title{
Study on characterization of cured adhesive by ultraviolet for encapsulation photovoltaic modules
}

\author{
Li Ling \\ Solar Energy Science and Engineering, Jiangxi Provincial Xinyu College, Xinyu, 336600, China \\ E-mail: 3073603237@qq.com
}

Keywords: UV-curing, Encapsulation, Solar cell modules, BIPV

\begin{abstract}
BIPV is the most important application of PV products, and BIPV solar modules will take a great share of the PV market in the future. BIPV solar modules which encapsulated with UV-cured adhesive is researched in this paper. The refractive index and dielectric constant of the UV curable adhesive films are measured and discussed. It is found that UV curable adhesive refractive index is 1.678 which is approximate with the toughened solar glass. The dielectric constant of UV-cured adhesive is 2.822, the quality of UV curable adhesive film is good with an ideal electricity insulation property. Result shows that this kind of modules encapsulated by UV-cured adhesive not only can absorb daylight fully, but also has a better electricity insulation property .UV-cured solar modules are suitable to be used in building.
\end{abstract}

\section{Introduction}

Building-Integrated Photovoltaic (BIPV) has been developed rapidly in the past decade. A number of BIPV projects can be found worldwide. BIPV technology has developed widely because of its characteristic which combines energy production with other functional featureson building façade [1]. Photovoltaic (PV) modules integrated into the building can cut down on costs and other resources. Solar glass curtain wall unit as an important symbol of modern architecture, has been widely used. The packaging format of solar glass curtain wall become the new market demand luminescent spot.

At present, EVA is also used in solar module, waterproofing treatments and seals. The sequence of EVA films is between modified epoxy resin and epoxy resin. However, due to structural characteristics of EVA itself, it easy brought yellowing and bonding strength is not enough. Our institute will be tend to use ultraviolet (UV)-cured BIPV modules to replace the traditional glass wall for energy efficiency and aesthetic consideration. New cured adhesive manufactured by our institute will be evaluated as improved packaging materials. This kind of solar modules encapsulated by UV-cured adhesive is composed of two layers of highly transparent glasssheets, and silicon solar cells which encapsulate by UV-cured adhesive. Figure 1 shows a simplified sketch of the cross-section of the UV-cured BIPV module. This kind of BIPV modules can generate electricity, at the same time, daylight can penetrate through the transparent adhesive between the solar cells. Therefor both decreasing material thermal resistance of the module and increasing temperature difference in cooling become very important.

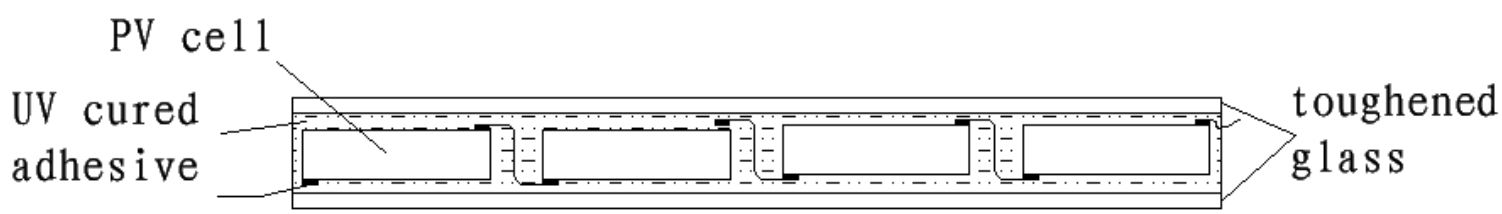

Figure 1. The structure of a UV-cured PV module (not to scale)

The UV cured adhesive provides the functions of (1) structural support, electrical and physical isolation, and thermal conduction for the solar cells and circuit components. (2) Opticalcoupling between the solar cells and the glass sheets. (3) Physical protection of the PV module from detrimental environmental factors. Chemical stabilization is often employed to enhance the 
durability of polymeric materials, especially for these used in integrated into the building.

To date, very few research has been conducted on the energy performance of the BIPV modules. Even a few studies on BIPV module can be found, there is no detailed study on the impact of encapsulated materials. In this paper characterization of UV-cured adhesive is analyzed in detail. The results of the refractive index and dielectric constant of the cured adhesive measured then discussed.

\section{Measurement and Test}

\subsection{Refractive Index}

As a new type of "Green Adhesive", UV-cured adhesive applied to encapsulated PV module. The refractive index of the UV-cured adhesive is a important characterization to indicate the encapsulated material of PV module. Therefore the refractive index of adhesive films was measured as a function to weigh how much daylight was aborted ${ }^{[2]}$.

Flow three kinds of liquid UV-cured adhesive down into the model box step by step, then cured by ultraviolet about 5 minutes until it has been cured completely. the area of adhesive films is $60 \mathrm{~mm}$ $\times 60 \mathrm{~mm}$, and the thickness is about $2 \mathrm{~mm}$. The refractive index of UV-cured adhesive films was measured in a way of fully refractive with Abbe refractometer. the refractive index of Abbe refractometer is within the range of $1.300 \sim 1.700$. Figure 1 shown the principle of the method of fully refractive.

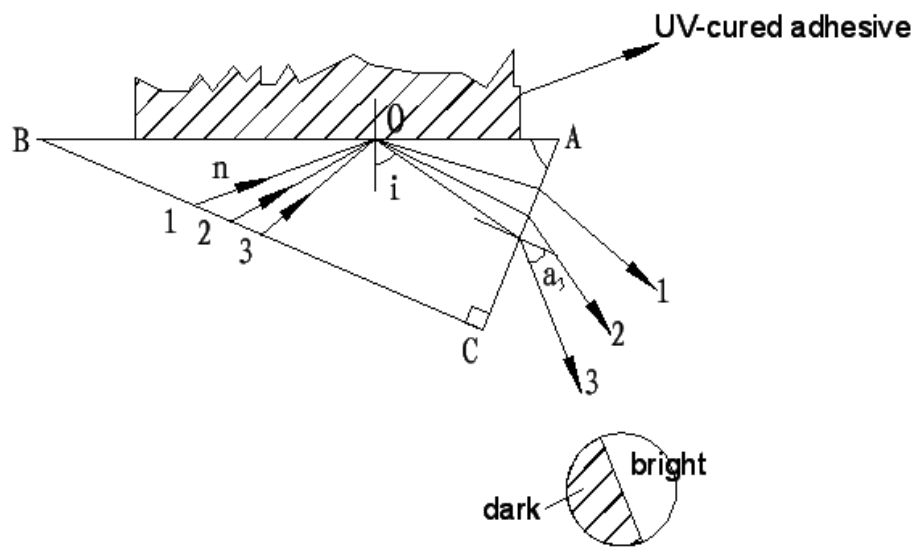

Figure 2. Abbe refractometer

As shown in table1. The refractive index of the UV-cured adhesive.

Table 1 Refractive index of the UV-cured adhesive

1 \# UV-cured adhesive

\begin{tabular}{|c|c|c|c|c|}
\hline & sample1 & sample 2 & sample 3 & average \\
\hline $\mathbf{n}_{\mathbf{x}}$ & 1.683 & 1.682 & 1. 680 & 1.682 \\
\hline \multicolumn{5}{|c|}{2 \# UV-cured adhesive } \\
\hline & sample 1 & sample 2 & sample 3 & average \\
\hline $\mathbf{n}_{\mathbf{x}}$ & 1.677 & 1.676 & 1.680 & 1.678 \\
\hline \multicolumn{5}{|c|}{3 \# UV-cured adhesive(anaerobic adhesive) } \\
\hline & sample 1 & sample 2 & sample 3 & average \\
\hline $\mathbf{n}_{\mathbf{x}}$ & 1.680 & 1.679 & 1.679 & 1.679 \\
\hline
\end{tabular}

Then with the same method to measure the refractive index of the toughened glass as frontsheet, and measured the thickness of the toughened glass with Micrometer, the results showed as follow: 
Table2 Refractive index of the toughened glass

\begin{tabular}{lll}
\hline sample & thickness $(\mathbf{m m})$ & refractive index \\
\hline toughened glass & 6.0 & 1.576 \\
\hline
\end{tabular}

\subsection{Dielectric Constant}

These are several kinds of polarization when the UV-cured adhesive at the electric field, such as(1)electron polarization,(2)atomic polarization,(3)dipole Polarization. $\varepsilon$ is the dielectric constant which can indicate the polarizability of UV-cured adhesive[3] .It is defined as follow:

$$
\varepsilon=\frac{C}{C_{0}}=\frac{Q / V}{Q_{0} / V}=\frac{Q}{Q_{0}}=\frac{Q+Q^{\prime}}{Q_{0}}
$$

In formula 1: where $\mathrm{V}$ is voltage between two plates of the capacitor; $\mathrm{Q}_{0}$, $\mathrm{Q}$ is the electric charge on the vacuum capacitor and UV-cured adhesive capacitor; $Q^{\prime}$ is induction Charge on the plates which polarize by UV-cured adhesive capacitor. From figure 1, we can see the dielectric constant reflects the ability of UV-cured adhesive capacitor to store the electric energy.

The instrument measured dielectric constant is the alternating current bridge QS18A. As showed in figure 3 , four arms $\tilde{Z}_{1}, \tilde{Z}_{2}, \tilde{Z}_{3}, \tilde{Z}_{4}$ is complex impedance, ab connect with alternating-current $E, c d$ connect with oscilloscope $D$.

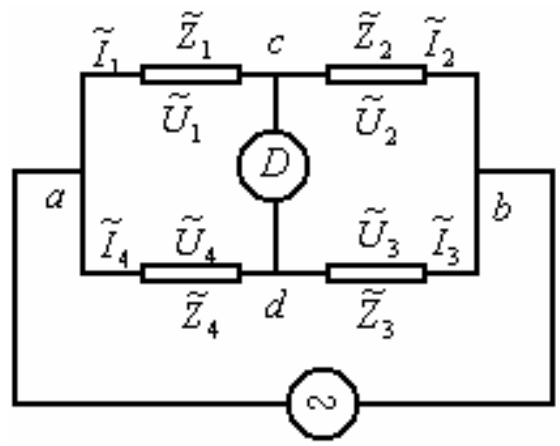

Figure 3 the balance of Alternating Current Bridge

When the alternating current bridge is balance, point $c$ and $d$ have the same electric potential. So we can get the condition of the balance Alternating Current Bridge:

$$
\tilde{Z}_{1} \tilde{Z}_{3}=\tilde{Z}_{2} \tilde{Z}_{4}
$$

The progress to measure unknown impedance $\tilde{Z}_{x}\left(\tilde{Z}_{x}=\tilde{Z}_{1}\right)$ with alternating current bridge just to make alternating current bridge balance. Generally speaking, $\tilde{Z}_{x}$ contains two unknown components. In fact, the condition of balance complex impedance means the condition of balance two real number, they both should balance firstly. To measure $\tilde{Z}_{x}$, at least there are two arms should be controlled in the Current bridge ,and all arms must depend on the balance of Alternating Current Bridge.

$\mathrm{C}_{4}$ is standard capacitor (missed the loss), R2, R3, R4 is non-inductance resistor, the balance condition is:

$$
\left(R_{x}-j \frac{1}{\omega L}\right) R_{3}=\left(R_{4}-j \frac{1}{\omega C}\right) R_{2}
$$

Make the real and imaginary equal: 


$$
C_{x}=\frac{R_{3}}{R_{2}} C_{4}, R_{x}=\frac{R_{4}}{R_{3}} R_{2}
$$

Cut two pieces of aluminum as the plates of the capacitor ,the area is $60 \mathrm{~mm} \times 60 \mathrm{~mm}$,Welded conducting wire with two plates of the capacitor, connected the plates showed as $C_{x}$ in figure3,take the adhesive samples from experiment 2.1 in two plates closely.

According to the basic principles of Alternating Current Bridge, at least there are two parameters should be controlled in the Current bridge ,when the two parameters balance ,the oscilloscope D showed zero. In fact, two parameters can't balance at the same time, so we must adjust one firstly, make the Instructions as small as possible, then adjust another. Adjust two arms again and again until the Instruction of the oscilloscope showed zero. From formula 4 we can get capacitance of the UV-cured adhesive.

Calculated as:

$$
\begin{gathered}
C_{4}=C_{0}=\frac{\varepsilon_{0} S}{d} \\
C_{x}=\varepsilon_{r} \bullet C_{0}
\end{gathered}
$$

Take formula 6 into formula 5:

$$
\begin{aligned}
& C_{x}=\frac{\varepsilon_{r} \varepsilon_{o} S}{d} \\
& \varepsilon=\varepsilon_{r} \varepsilon_{0}
\end{aligned}
$$

Calculated as:

$$
\varepsilon=\frac{C_{x} d}{S}
$$

From formula 9 we can calculate the dielectric constant of UV cured adhesive. The results showed as table 3 .

Where $\varepsilon_{0}=8.85 \times 10^{-12} \mathrm{C}^{2} /\left(\mathrm{N} \bullet \mathrm{m}^{2}\right), S=60 \mathrm{~mm} \times 60 \mathrm{~mm}=3.6 \times 10^{-3}, \quad k=9.0 \times 10^{9} \mathrm{~N} \bullet \mathrm{m}^{2} / \mathrm{C}^{2}$.

Table 3 the dielectric constant of UV cured adhesive

\begin{tabular}{lllll}
\hline sample & thickness $d(\mathbf{m})$ & capacitor $C_{x}(\mathbf{F})$ & $\begin{array}{l}\text { dielectric constant } \\
\varepsilon_{r}\end{array}$ & $\begin{array}{l}\text { Dielectric constant } \\
\varepsilon\left[C^{2} /\left(N \cdot m^{2}\right)\right]\end{array}$ \\
\hline $\mathbf{1} \#$ & $3 \times 10^{-3}$ & $3.008 \times 10^{-11}$ & 2.832 & $2.507 \times 10^{-11}$ \\
$\mathbf{2} \#$ & $2.8 \times 10^{-3}$ & $3.203 \times 10^{-11}$ & 2.815 & $2.491 \times 10^{-11}$ \\
$\mathbf{3} \#$ & $2.4 \times 10^{-3}$ & $3.744 \times 10^{-11}$ & 2.820 & $2.496 \times 10^{-11}$ \\
\hline
\end{tabular}

\section{Discussion}

As shown in Table 1 and Table 2 the refractive index of the UV-cured adhesive and toughened glass, the results of refractive index is so approximate. When the daylight shine on the PV module encapsulated by UV-cured adhesive, this kind of encapsulate material can cut down the albedo of PV module, enhance the photoelectric conversion efficiency of PV module.

Formula derived from the classical theory of photoelectric absorption as follow ${ }^{[4]}$ :

$$
n^{2}=\frac{1}{2} \varepsilon_{r}\left[1+\left(1+\frac{\sigma^{2}}{\omega^{2} \varepsilon_{r}^{2} \varepsilon_{0}^{2}}\right)^{1 / 2}\right]
$$

Where $n$ is refractive index, $\varepsilon_{r}$ is dielectric constant of UV-cured adhesive, $\varepsilon_{o}$ is vacuum 
dielectric constant, $\sigma$ is UV-cured adhesive conductivity, $\omega$ is angular frequency, from formula 10 ,we can see $n \approx \sqrt{\varepsilon_{r}}, \sigma \approx 0$.from figure 1 and figure 2,we calculate $n \approx \sqrt{\varepsilon_{r}}, \sigma \approx 0$, so we can know UV-cured adhesive is a good insulate material.

Non-polar molecule only has atomic polarization and electron polarization, $\varepsilon$ is lower; polar molecule has dipole polarization except for the two polarization above mentioned, $\varepsilon$ is higher. In addition to there are some factors focus on the dielectric constant $\varepsilon$ :

(1) Symmetric structure of the molecular. When the molecule is Symmetrical, the polarity will be offset each other completely or partially.

(2) The force of molecular. Increased molecular force (cross-linked, orientation, crystal) will make $\varepsilon$ higher .decrease molecular force would make $\varepsilon$ lower.

(3) Physical state. The High-elastic polar groups have a better orientation than glass-state polar groups, so $\varepsilon$ is higher.

Three kinds of average dielectric constant of UV-cured adhesive is 2.822. all above, we can increase the force of UV-cured adhesive between toughened glasses to enhance the dielectric constant, and improve its electrical insulation.

\section{Conclusions}

Building integrated photovoltaic (BIPV) perform traditional architectural functions of wallsand roofs while also generating electricity. Compared with normal buildings, BIPV reduces the air conditioning load caused by indoor heat gain through the wall and improves building energy efficiency. The displacement of utility generated electricity. A method of preparing packaging UV-cured adhesive was introduced and the main factors to influence its performances were analyzed. It is found that UV curable adhesive refractive index is 1.678 which is approximate with the foresheet glass. The dielectric constant of UV-cured adhesive is 2.822, the quality of UV curable adhesive films were good with an ideal electricity insulation property.

Finally, results showed that this kind of modules encapsulated by UV-cured adhesive cannot only absorptive daylight fully, but also has a better electricity insulation property .UV-cured solar cell modules are suitable to be used in building.

\section{Acknowledgement}

The fund comes from Jiangxi Province Department of Education GJJ 151205.

\section{References}

[1] A.W. Czanderna and F.J. Pern. Encapsulation of PV Modules using Ethylene Vinyl Acetate Copolymer as a Pottant: A Critical Review. Solar Energy Materials and Solar Cells, America, 1996(43):101-183. (For a paper in journal)

[2] Wu Qiang and Guo Guangcan. opto-electronics. China, 1996. (For a book)

[3] Huang Xiang, li Zhuang. Electron optics. China, 1998. (For a book)

[4] WHIPPLE C L, THOHE J A. Performance of elastomeric sili-cones in ablative and space environments, Rubber Chem. And Technol., 1966, 39(1):247. (For a paper in journal) 